\title{
The Relationship Between Radiographic and Anthropomorphic Measurements of Deformity of the Thorax, Hips, and Pelvis in Adults with Cerebral Palsy
}

Carlee Holmes MPhysio (Neuro)

St. Vincent's Hospital, Young Adult Complex Disability Service, Health Independence Programs, Melbourne, Australia; Physiotherapy Department, Monash University, Frankston, Australia

Kim Brock $P h D$

St. Vincent's Hospital, Physiotherapy Department, Melbourne, Australia

Prue Morgan PhD

Physiotherapy Department, Monash University, Frankston, Australia

\section{ABSTRACT}

Non-ambulant adults with cerebral palsy (CP) are commonly affected by progressive secondary debilitating musculoskeletal issues, which may be clinically measured using the Goldsmith Indices of Body Symmetry (GlofBS). The primary aim of this study was to explore relationships between clinical outcomes and relevant radiographic measures in non-ambulant adults with CP. Thoracic shape and symmetry, pelvic orientation, and hip range were measured using the GlofBS. Radiographs of the pelvis and spine were reviewed. The Pearson correlation ( $r$ ) or Pearson's $r$ was used to assess the relationships between clinical and radiographic measures. The positioning and readability of radiographic data in 30 non-ambulant adults with CP were variable. Minimal to no correlation between paired measures of radiographic and clinical data for trunk, pelvis, and hips were found, ranging from the lowest correlation of $r(15)=-0.09, p=0.620$ (left migration percentage and hip range) to the highest of $r(15)=-0.25, p=0.200$ (right hip morphology scale and hip range). The complex three-dimensional nature of asymmetries of the thorax, pelvis, and hips, measured clinically with the GlofBS, provides valuable, yet different, postural information to that obtained by radiographs. Inclusion of both radiographs and the GlofBS would enable a comprehensive lifespan assessment for postural management of adults with CP.

Holmes, C., Brock, K. \& Morgan, P. (2021). The relationship between radiographic and anthropomorphic measurements of deformity of the thorax, hips, and pelvis in adults with cerebral palsy. New Zealand Journal of Physiotherapy, 49(1), 15-23. https://doi.org/10.15619/NZJP/49.1.03

Key Words: Cerebral Palsy, Posture, Radiograph, Scoliosis, Windswept Hips

\section{INTRODUCTION}

The secondary musculoskeletal consequences of cerebral palsy $(\mathrm{CP})$, a permanent, life-long condition acquired before, during, or after birth, are progressive and often debilitating. The arising postural asymmetries affecting the spine, pelvis, and hips may result in further adverse consequences, such as pain and pressure injuries (Gudjonsdottir \& Mercer, 1997), especially for nonambulant adults with CP. The risk of mortality due to respiratory disease in adults with CP is much greater than the general population (Ryan et al., 2019), with postural asymmetry of the thoracic cage contributing to increased risk in the most severely affected adults (Horimoto et al., 2012). Postural asymmetry involving limited hip flexion, pelvic obliquity, trunk asymmetry, scoliosis, and windswept hip posture is common in adults with CP with low motor function (Ágústsson et al., 2018), described on the Gross Motor Function Classification System (GMFCS) as levels IV and V (Palisano, et al., 1997). These asymmetries typically occur alongside pain and spasticity, further adversely affecting function and participation (Benner et al., 2019).

The identification, monitoring, and management of secondary postural complications for adults with CP is critical, given the impact on many domains of health and functioning alongside carer burden and economic impacts. The specific requirements for non-ambulant adults with $\mathrm{CP}$ results in the need for specialised equipment, support workers, hospital admissions and reliance on crisis services (Collis et al., 2008; Gudjonsdottir \& Mercer, 1997). This presents particular challenges for non-ambulant adults with CP who frequently have limitations in communication and cognition. Despite their potential importance, there is a lack of standardised measurement techniques beyond radiographs to record postural asymmetry in this population (Benner et al., 2019; Holmes et al., 2018). In addition, capturing the complex three-dimensional asymmetry of the thoracic cage and windswept hips with a reliable measurement tool can prove even more challenging. Physiotherapists are well placed to fill this critical surveillance role within both standard and advanced scope of practice roles (World Physiotherapy, 2019).

When radiographic surveillance is possible, there are limitations in interpretation of objective findings for those with significant postural asymmetry. The Cobb angle and migration percentage (MP) are recommended radiographic measurements used to assess status of scoliosis and hip displacement respectively in those with CP. The Cobb angle is a radiographic objective 
measure of the extent of spinal curvature (Cobb, 1948), reported in degrees, with scoliosis defined as a Cobb angle $\geq 10^{\circ}$ (Oda et al., 2017). The MP is a radiographic measure (in percentage form) of the amount of ossified femoral head not covered by the ossified acetabulum (Reimers, 1980). The Australian Hip Surveillance Guidelines considers MP of $\leq 10 \%$ to be normal and MP $\geq 30 \%$ as abnormal or "at risk" (Wynter et al., 2014). The Cobb angle and MP represent two-dimensional measures of complex three-dimensional skeletal deformities, and may be limited in effectively documenting functional postural deformity. The rotary components of thoracic asymmetry and windswept hips are thus difficult to ascertain with radiographic studies alone in non-ambulant adults with CP. Due to the potential for progression of scoliosis and hip displacement in this vulnerable population, and the aforementioned limitations with radiographic monitoring, there is a need for an additional valid and reliable clinical measurement tool that can capture three-dimensional elements of posture to be used alongside radiographs to assist in functional management.

The Goldsmith Indices of Body Symmetry (GlofBS) is a clinical measurement tool providing a systematic, objective and threedimensional approach for the identification of asymmetry of the chest, pelvis, and hips (Goldsmith et al., 1992). The chest measurements provide a component of axial rotation and the hip and pelvic measurements occur across more than one plane of movement, as compared to the views obtained from plain radiographs and goniometer measures, which only provide anterior/posterior or lateral measures. The GlofBS was chosen as a clinical measurement tool potentially suited for adults with severe neuromuscular disability, due to the scarcity of alternative tools that can reliably capture three-dimensional aspects of complex postural asymmetry in this population. The constructs captured with the GlofBS have noted relevance to seated and sleeping positions, impacting on pain, pressure risks, and function. Satisfactory intra- and inter-rater reliability of the GlofBS have recently been determined in non-ambulant adults with $C P$, and control data have been established (Holmes et al., 2020).

Exploration of the relationships between radiographic and anthropomorphic measurements of postural deformity in adults with CP may provide valuable information to assist clinical management of adults with complex disabilities, and suggest effective assessment tools to identify specific elements of postural asymmetry. Continuity of care and specialist knowledge are two of the identified barriers to effective transitioning of young adults from paediatric to adult healthcare services (Burns et al., 2014), which may be addressed with use of the GlofBS across the lifespan.

The objectives of this study were to establish the relationship between radiographic and anthropomorphic measurements of postural deformity in adults with $C P$, such as described with the GlofBS. Specifically, this study aimed to explore any relationship between GlofBS measures and radiographs in adults with $C P$ classified as GMFCS levels IV or V.

\section{METHODS}

This cross-sectional study used data arising from 30 adults with $\mathrm{CP}$, some of whom participated in a measurement tool reliability study to undertake secondary analysis of previously unreported radiographic data (Holmes et al., 2020).

\section{Participants and setting}

All patients referred to the Young Adult Complex Disability Service between February 2017 and December 2018 were considered for inclusion. Patients with a diagnosis of CP classified as GMFCS level IV or $V$ were eligible for inclusion in the study. Patients were excluded if they had a severe movement disorder or behavioural issues that placed either themselves or the raters at risk during measurement, or if they were pregnant. Ethical approval was gained from St Vincent's Hospital, Melbourne, Human Research Ethics Committee (HREC/16/ SVHM/148). All participants (or their next of kin) signed informed consent forms.

\section{Outcome measures}

\section{GlofBS}

As per standard testing protocol, the relevant GlofBS outcome measures collected for analysis were (a) chest right left ratio (indicative of chest rotation), (b) combined hip external rotation/ abduction (left and right), and (c) the Windswept Index (indicative of the degree of asymmetry between the left and right pelvis/lower limbs) (Goldsmith et al., 1992; Goldsmith \& Goldsmith, 2013). These measures have previously been shown to have excellent inter- and intra-rater reliability in this population (Holmes et al., 2020). The components of the GlofBS anatomical measurement instrument are illustrated in Figure 1, with further illustrations of the measurement process provided in Figure 2.

\section{Figure 1}

Anatomical Measurement Instrument

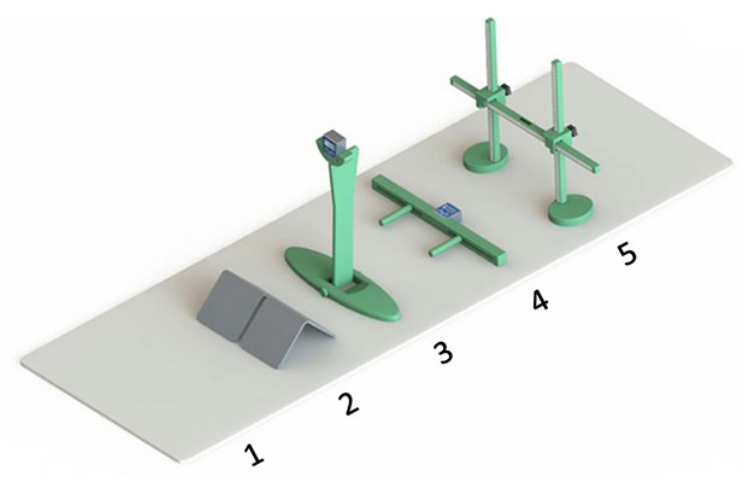

Note. Anatomical measurement instrument with equipment listed from left to right: $1=$ non-slip mat; 2 = foot brackets; 3 = leg paddle with level box angle sensor; 4 = pelvic bridge with level box angle sensor; $5=$ chest frame (Goldsmith \& Goldsmith, 2013).

\section{Hip/spine radiographs}

Antero-posterior (AP) radiographs of the pelvis and spine obtained within 12 months of the collection of the GlofBS data were reviewed. The Australian Hip Surveillance Guidelines for Children with Cerebral Palsy recommend surveillance every 12 months beyond skeletal maturity in the presence of abnormal MP, progressive scoliosis, or significant pelvic obliquity (Wynter et al., 2014), thus a 12-month time frame was considered acceptable for this study. All radiographic measurements were completed using tools within a picture archiving and 
Figure 2

Goldsmith Indices of Body Symmetry Measurement Process
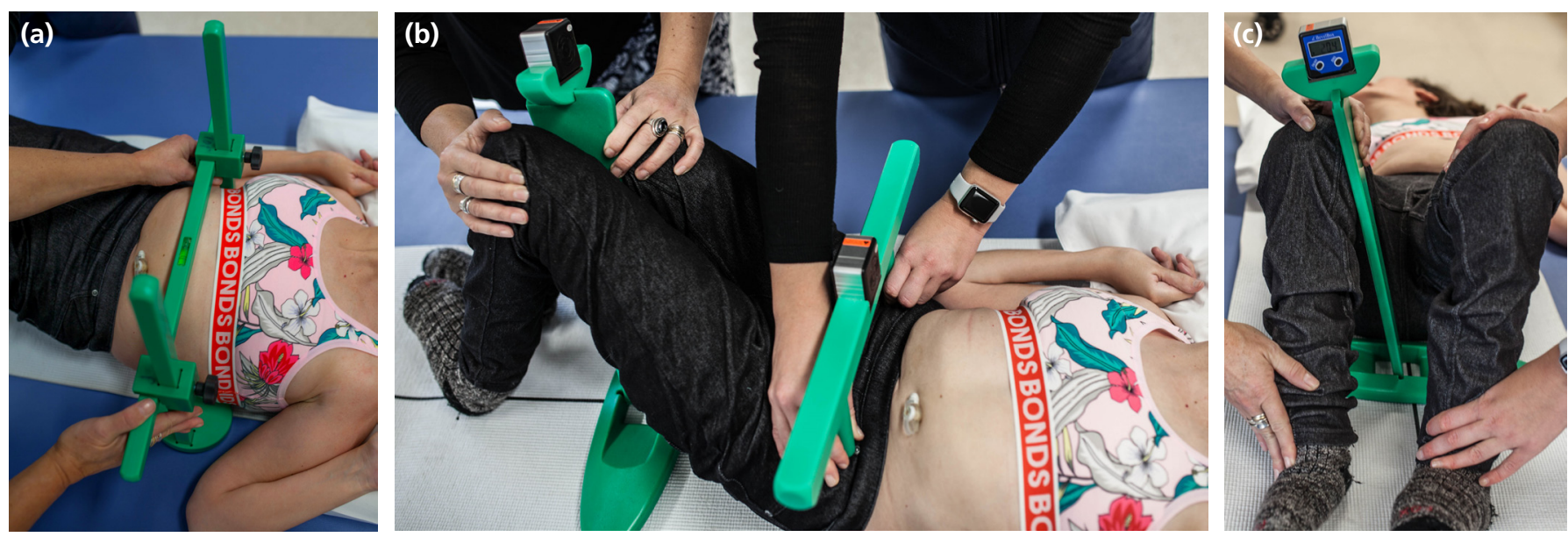

Note. Depiction of the Goldsmith Indices of Body Symmetry measurement processes: (a) chest frame to gain chest right left ratio and chest depth width ratio; (b) pelvic bridge and leg paddle to gain measures used to calculate Windswept Index; (c) leg paddle to gain right hip external rotation/ abduction.

communication system (PACS) (Synapse ${ }^{\mathrm{TM}}$, Fujifilm Corp., Tokyo, Japan).

\section{Procedures}

GlofBS measurements were performed by an experienced therapist (rater) having undergone additional training in administration of the GlofBS Anatomical Measurement Indices as per a standard testing protocol (Goldsmith \& Goldsmith, 2013). A testing session typically lasted $45 \mathrm{~min}$.

Radiographic evaluation and measurement for each participant was undertaken by a senior orthopaedic physiotherapist, who is a postgraduate research fellow with 10 years' experience, and responsible for radiographic evaluation and measurement in a clinical musculoskeletal surveillance service. Measurement of MP, Cobb angle and pelvic obliquity, and grading of hip status according to the Melbourne Cerebral Palsy Hip Classification Scale (MCPHCS) (Robin et al., 2009) were undertaken. The MCPHCS is a six-level radiographic ordinal scale used to classify morphology of the skeletally mature hip. The classification covers a wide range of radiographic features, from Grade I (normal hip) through to Grade V (dislocated hip) and Grade VI (dislocated hip that required salvage surgery). The classification includes sub-classifications for femoral head deformity, acetabular deformity, and pelvic obliquity (Robin et al., 2009; Shrader et al., 2017). Pelvic obliquity was measured as the angle between the inter-teardrop line and a horizontal reference line parallel to the frame of the radiograph (Heidt et al., 2015). If the inter-teardrop line was obscured by gonadal shielding, the inter-ischial or inter-iliac crest line was used (Heidt et al., 2015). A quality rating was provided for each radiograph (not readable/ readable/challenging to read), and the position in which the spine radiograph was obtained was recorded (supine/sitting/ not reported). Any limitations in evaluating the radiograph and obtaining valid measurements were recorded, along with any reason for missing data (e.g. inadequate participant position invalidating measurement). If more than one spinal curve was present, the largest Cobb angle was selected. The highest MP (left or right hip) was noted. The researcher was blinded to GlofBS results for each participant.

The following paired data were selected for exploration of any relationship between GlofBS and radiographic measures based on similarity of investigation of a specific skeletal area (i.e. spine, hip).

1. Chest right left ratio compared to Cobb angle (largest angle if $>1$ curve).

2. Combined hip external rotation and abduction compared to MP (right and left).

3. Combined hip external rotation and abduction compared to the MCPHCS (right and left).

4. Windswept Index compared to the highest MP (either right or left).

\section{Statistical analyses}

The SPSS statistical software version 24 (SPSS Inc., Chicago, Illinois) was used for all quantitative analyses. Normality of all data was evaluated using visual inspection of the histograms and evaluation of the Kolmogorov-Smirnov statistic, with $p$ $>0.05$ satisfying normal distribution. Mean scores and SDs for each variable were calculated once normative data was confirmed. The following adjustments to the data were made if required to facilitate analyses. The GlofBS chest right-left ratio was adjusted to reflect the magnitude of the measure rather than a positive or negative value (indicative of rotation in a clockwise or anticlockwise direction). The Windswept Index was adjusted to reflect absolute values rather than positive and negative values either side of zero. 
Parametric analysis was undertaken using Pearson's correlation $(r)$ to assess correlation between paired GlofBS outcomes against radiographic measures (Cobb angle, MP and MCPHCS). The strength and direction of any relationships $(r)$ were established according to Cohen (1988), where 0.1 to $0.29=$ small, 0.30 to $0.49=$ medium and 0.50 to $1.0=$ large effect size. Significance was set at $p<0.05$.

Scatterplots were constructed to visualise relationships between GlofBS variables and key radiographic data. Individual cases were identified that were outside the cut-offs previously established from the literature for the Cobb angle and MP (Oda et al., 2017; Wynter et al., 2014). For GlofBS measures, an a priori decision was made to use control data to calculate cut-off cases sitting above or below 2 SD (95\% of cases) from the mean (Holmes et al., 2020), described as very high or very low scores.

\section{RESULTS}

After screening 165 potential participants, 30 adults participated in the study, with a median age of 19 years (range 17-38). Of the 135 excluded, 36 declined, 34 did not have a diagnosis of CP, 46 did not have function classified at GMFCS level IV or $\mathrm{V}$, five had a severe movement disorder (dystonia), nine had significant cognitive/behavioural challenges, and five did not have radiographs available. Of the 30 participants, 29 had CP sub-type quadriplegia and one had diplegia, 10 had received previous surgery for scoliosis, 20 had no consistent communication methods, two used communication devices, and eight were verbal communicators.

Positioning for spinal radiographs was variable: nine of 30 spinal radiographs were performed in a supine position and 13 in a sitting position, while six did not have a documented position; two participants were missing spinal radiographs. The quality of the spinal radiographs also varied, with five of 30 not readable and five reported as challenging to read. Only two of the 30 hip radiographs were not readable in a valid and reliable manner due to extreme positioning of the hip into fixed abduction and abduction/external rotation (Figure 3).

Demographic and postural data for the cohort are presented in Tables 1 and 2 . The total number of participants included in the spinal and hip radiograph data was 28 and 30, respectively. Of note, from the radiographs, 10 hips were reported as "at risk", eight hips were considered within normal limits (MP $\leq 10 \%), 19$ participants had a documented scoliosis, and 27 participants had pelvic obliquity. Cobb angles as high as $93^{\circ}$ were identified, and pelvic obliquity ranged from $0^{\circ}$ (two participants) to $29^{\circ}$ (two participants). The majority of hips (15 right 16 left) were described as Grade III using the MCPHCS (Table 2).

\section{Table 1}

\section{Participant Demographics}

\begin{tabular}{lc}
\hline Demographic & $n^{\text {a }}$ \\
\hline Gender & \\
Male & 17 \\
Female & 13 \\
Age (years) & Median 19, range 17-38 \\
GMFCS level & 3 \\
IV & 27 \\
V & \\
\hline
\end{tabular}

Note. GMFCS = Gross Motor Function Classification System.

a Except where indicated

Any association between radiographic and GlofBS outcomes for trunk, pelvis, and hips was explored. Minimal to no correlation between paired measures of radiographic and GlofBS data was found ranging from lowest correlation: $r(15)=-0.09, p=0.620$ (left MP and left external rotation/abduction) to highest: $r$ (15) $=-0.25, p=0.200$ (right MCPHCS and right external rotation/ abduction) (Table 3).

Scatterplots were constructed to illustrate the relationship between the Windswept Index and highest MP (Figure 4), right MP and right abduction/external rotation (Figure 5), and the highest Cobb angle and chest right left ratio (Figure 6). Scatterplots of these comparisons demonstrate that the majority of participants had Cobb angles and MP values above control cut-off values. In comparison, for GlofBS values, more people with CP fell within the control range (+/- 2 SD).

\section{Figure 3}

Example of Radiographic View of Pelvis and Hips From Which Migration Percentage is Calculated

Note. Right hip migration percentage: 100\%. Left hip migration percentage: unable to complete valid and reliable assessment and measurement (Reimers, 1980) due to hip positioning in extreme abduction and external rotation.
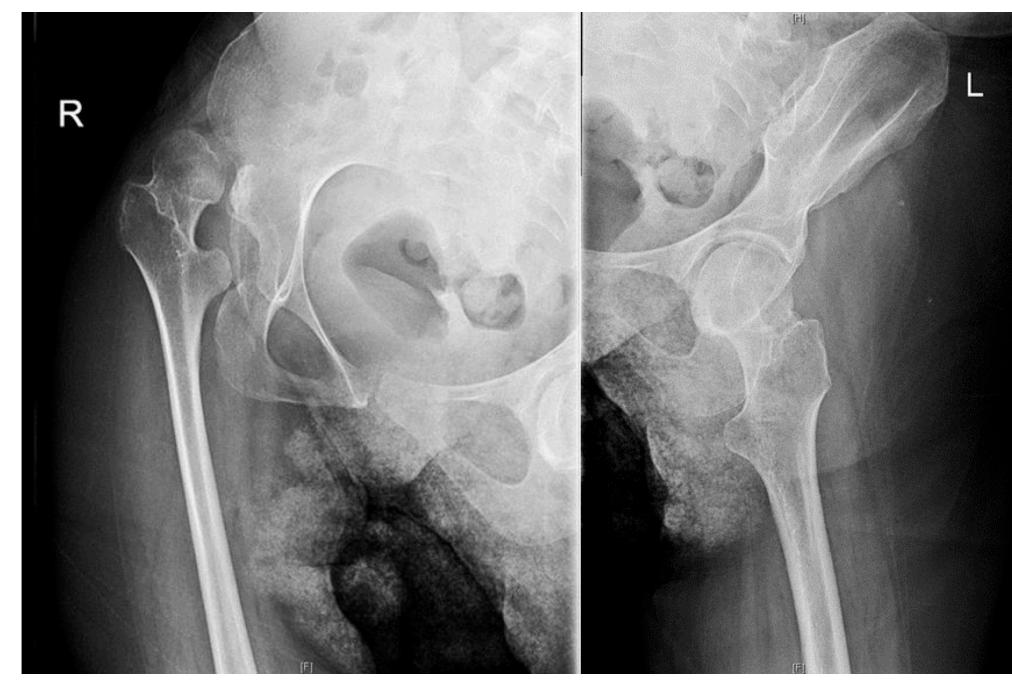
Table 2

Participant Postural Data

\begin{tabular}{|c|c|c|}
\hline GlofBS a & Mean (SD) & Range \\
\hline Chest right left ratio (magnitude) & $0.16(0.13)$ & $0.01-0.56$ \\
\hline Windswept Index (absolute values) & $19.02(22.74)$ & $0.75-81.00$ \\
\hline Right external rotation/abduction $\left(^{\circ}\right)$ & $43.79(19.57)$ & $7.80-79.00$ \\
\hline Left external rotation/abduction $\left(^{\circ}\right)$ & $46.19(16.43)$ & $2.25-70.00$ \\
\hline Radiographic data & Mean (SD) Range & Incidence, $n$ \\
\hline Highest migration percentage (\%) ${ }^{b}$ & $\begin{array}{l}31(22) \\
0-100\end{array}$ & $\begin{array}{c}<10,8 \\
10-30,38 \\
31-99,8 \\
100,2\end{array}$ \\
\hline Largest Cobb angle $\left(^{\circ}\right)^{c}$ & $\begin{array}{c}32(24) \\
7-93\end{array}$ & $\begin{array}{c}<10,3 \\
10-30,10 \\
30-60,6 \\
>60,3\end{array}$ \\
\hline Pelvic obliquity $\left({ }^{\circ}\right)^{d}$ & $\begin{array}{l}8(9) \\
0-29\end{array}$ & $\begin{array}{c}<10,21 \\
11-20,5 \\
21-30,3\end{array}$ \\
\hline
\end{tabular}

\begin{tabular}{lc}
\hline MCPHCS e & Right/left hip, $n$ \\
\hline Grade I & $2 / 0$ \\
Grade II & $6 / 7$ \\
Grade III & $15 / 16$ \\
Grade IV & $3 / 4$ \\
Grade V & $1 /$ \\
Grade VI & $1 / 0$ \\
\hline
\end{tabular}

Note. GlofBS = Goldsmith Indices of Body Symmetry; MCPHCS = Melbourne Cerebral Palsy Hip Classification Scale.

${ }^{\mathrm{a}} n=60 ; 4$ unreadable quality, ${ }^{\mathrm{b}} n=2$ missing spinal radiographs, $n=6$ unreadable quality; ${ }^{\mathrm{c}} n=1$ unreadable quality; ${ }^{\mathrm{d}} 2$ participants unable to be graded due to poor radiograph quality.

Control data for comparison: Mean (SD), range: Chest right left ratio (magnitude): 0.07 (0.05), 0.00-0.27; Windswept Index (absolute values): 3.59 (3.21), 0.00-14.25; Right external rotation/abduction $53.61^{\circ}\left(6.19^{\circ}\right)$, 35.00 $-65.50^{\circ}$; Left external rotation/abduction $55.79^{\circ}\left(6.84^{\circ}\right), 41.75^{\circ}-69.00^{\circ}$ (Holmes et al., 2020).

\section{Table 3}

Correlations between Goldsmith Indices of Body Symmetry and Radiographic Data

\begin{tabular}{lcc}
\hline Variable & Pearson's $r$ & $p$ \\
\hline Cobb angle and chest right left ratio & 0.13 & 0.580 \\
Left MP and left external rotation/abduction & -0.09 & 0.620 \\
Right MP and right external rotation/abduction & -0.19 & 0.330 \\
MP and Windswept Index & -0.23 & 0.240 \\
Right MCPHCS and right external rotation/abduction & -0.25 & 0.200 \\
Left MCPHCS and left external rotation/abduction & -0.19 & 0.350 \\
\hline
\end{tabular}

Note. $\mathrm{MCPHCS}=$ Melbourne Cerebral Palsy Hip Classification Scale; MP = migration percentage. 


\section{Figure 4}

Scatter Plot Depicting the Relationship Between the Windswept Index and the Highest Migration Percentage

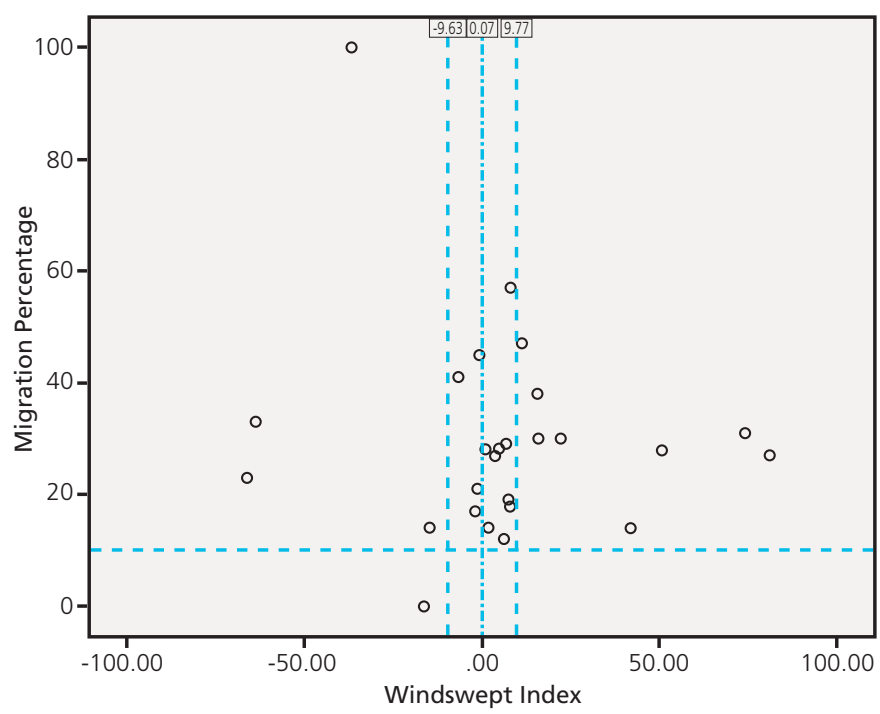

Note. The vertical dotted lines represent the mean \pm 2 SD. The horizontal dots represent $10^{\circ}$ migration percentage.

Figure 4 depicts the relationship between the Windswept Index and the highest MP. It illustrates that 28 of 29 participants had an MP of concern $\left(M P \geq 10^{\circ}\right.$ ), yet only 12 participants had a Windswept Index of concern (outside 2 SD of control data).

\section{Figure 5}

Scatter Plot Depicting the Relationship Between the Right Migration Percentage and Right Hip External Rotation/Abduction

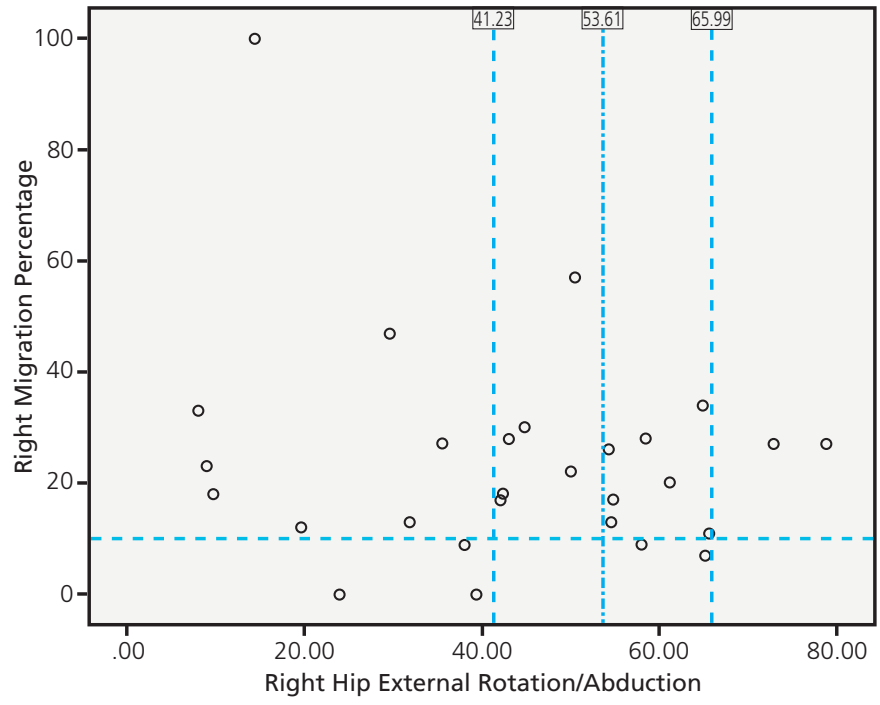

Note. The vertical dotted lines represent the mean \pm 2 SD. The horizontal dots represent $10^{\circ}$ migration percentage.

Figure 5 depicts the relationship between right MP and right hip external rotation/abduction. It illustrates that 23 of 28 participants had a right MP of concern $\left(M P \geq 10^{\circ}\right)$, yet only
10 of these also had a hip range of concern (outside 2 SD of control data).

\section{Figure 6}

Scatter Plot Depicting the Relationship Between the Highest Cobb Angle and Chest Right Left Ratio

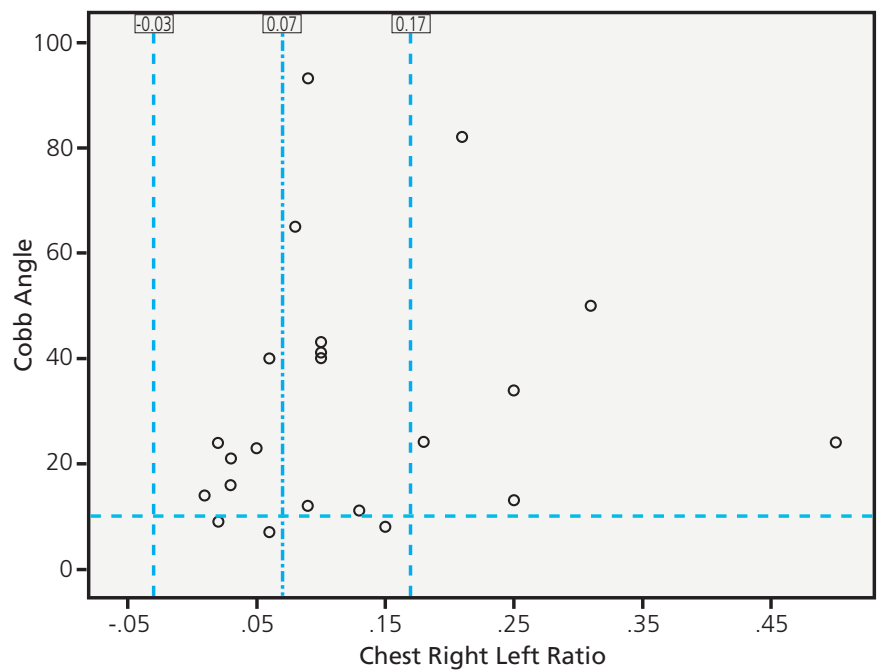

Note. The vertical dotted lines represent the mean \pm 2 SD. The horizontal dots represent $10^{\circ} \mathrm{Cobb}$ angle.

Figure 6 depicts the relationship between the highest Cobb angle and the chest right left ratio. It illustrates that while 19 participants had a Cobb angle of concern $\left(\geq 10^{\circ}\right)$ only six also had a chest right left ratio of concern (outside 2 SD of normative data).

\section{DISCUSSION}

This study is the first to explore relationships between an anthropometric measurement tool (GlofBS) for thoracic shape and symmetry, pelvis, and hips, and similar radiographic measures in a sample of non-ambulant young adults with $C P$ attending a large metropolitan healthcare service. As found in a previous study (Holmes et al., 2018), the incidence of hip displacement and scoliosis was high, with 19 of 22 participants demonstrating a scoliosis $\geq 10^{\circ}$ and 48 hips demonstrating an $\mathrm{MP} \geq 10^{\circ}$. All correlations between paired GlofBS variables and radiograph outcomes were small and not significant, suggesting that these tools measure two different constructs.

The study findings highlighted that measures of the Windswept Index alone (using the GlofBS) cannot be used to predict the presence or absence of hip displacement (MP). Similarly, hip MP is not related to hip mobility range in this cohort, as those with higher MP values demonstrated hip range values both higher and lower than hip external rotation/abduction range observed in control comparisons. The radiographic MCPHCS also bore minimal relationship to anthropometric constructs as measured with the GlofBS. Previous studies have also found that physical examination of joint range of motion via goniometry cannot replace information gleaned from radiographs in children with CP (Hägglund et al., 2007; Pruszczynski et al., 2016; Soo et al., 2006), as physical examination of hip range in a paediatric study was a poor indicator of risk of hip displacement (Hägglund 
et al., 2007). In this study, excess hip movement range was typically unilateral, associated with windswept hips and fixed postural deformity, often of an extreme nature in most participants. It is therefore not surprising that the MCPHCS and the GlofBS hip external rotation/abduction showed little relationship considering the MCPHCS is used to describe hip morphology as opposed to the GlofBS measure of hip mobility (GlofBS hip external rotation/abduction).

GlofBS measures of chest asymmetry (rotation) in this study also showed little correlation with radiographic measures of Cobb angles, suggesting the GlofBS is measuring a different spinal construct. Only six of 22 participants demonstrated both clinically significant Cobb angles (scoliosis) and extreme chest asymmetry. Previous studies exploring parameters correlated with the Cobb angle have demonstrated varying results (Sato et al., 2016; Suzuki et al., 1993), possibly indicative of the complex nature of the thoracic cage deformity. For example, three-dimensional aspects of scoliosis involving lateral deviation and spinal rotation measured with ultrasound were found to have only a small (but significant) correlation to the Cobb angle in a cohort of 11 children with CP (Suzuki et al., 1993). Frequent documentation of the three-dimensional nature of the rotational postural deformity of the thoracic cage will thus require additional clinical tools beyond radiographs and ultrasound, such as the GlofBS, to provide a comprehensive understanding of the stability of the thoracic asymmetry and any responsiveness to interventions.

A recent scoping review on postural asymmetry in adults with $\mathrm{CP}$ noted that non-standardisation of radiographic positioning is common in this population (Holmes et al., 2018). The Australian Hip Surveillance Guidelines for Children with Cerebral Palsy (Wynter et al., 2014) recommend a standardised position for AP pelvis radiographs (supine with neutral pelvic tilt, and neutral hip rotation and abduction), yet this is not always achievable. As noted in this study, the feasibility of obtaining a standardised position for imaging is often compromised in the complex adult CP population due to contracture, and cognitive and movement disorder challenges. In this study, the spinal radiograph positioning was variable with supine recorded for nine and sitting recorded for 13 participants. The Cobb angles from five spinal radiographs were unable to be measured at all, and five were noted to be challenging to accurately measure because of image quality issues. Positioning of participants for AP pelvis radiographs was similarly variable, decreasing validity of an MP measurement on some radiographs. A valid MP measurement was unable to be obtained at all for either hip for two of the participants due to significant windswept positioning for one and wide hip abduction positioning ("frog leg" image) for another. Error may occur in radiographs due to positioning error and/or measurement error (Schmid et al., 2016), with measurement error +/- 5\% for MP measurement (Schmid et al., 2016) and $4 \%$ to $8 \%$ for Cobb angle measurement (Gstoettner et al., 2007). The positioning error is largely unknown, with suggestions that it may be up to 30\% (Schmid et al., 2016). Mandatory recording of variance from the standard radiographic position for hip surveillance and spinal monitoring would facilitate greater accuracy in ongoing management of adults with $C P$, as the required quality and accuracy of radiographs for standardised and accurate measurement is not always possible in this population. An additional measurement tool with established reliability (Holmes et al., 2020), such as the GlofBS, would be of value.

A greater understanding of the observed rotary postural elements of the spine and hip/pelvis can be gleaned with the addition of the GlofBS to appropriate radiographic studies where feasible, adding to optimal functional management of non-ambulant adults with CP. This enables treating therapists to regularly monitor effectiveness of non-surgical interventions, such as tailored sleep systems (Public Health England, 2018). There is also the potential to use the GlofBS to monitor postural asymmetry in other populations with significant neuromuscular dysfunction, such as muscular dystrophy or multiple sclerosis. However, measurement of asymmetry of the spine, hips, and pelvis remains particularly challenging for those patients with severe cognitive or extreme movement disorders who may not tolerate either radiographs or bedside measurement using GlofBS. Further research is required in this area to explore the potential for digital photography monitoring or shape capture methods, such as three-dimensional laser scanning systems for fabrication of customised seating systems (Tasker et al., 2011) or dual energy x-ray absorptiometry, commonly used in athletic populations (Nana et al., 2016) and for those with eating disorders (Stewart et al., 2012) to track changes in body composition. Biomedical imaging, despite its many diagnostic, prognostic, and therapeutic applications (Farahani et al., 2017), remains unexplored in the measurement of postural asymmetry.

With the recent introduction of the National Disability Insurance Agency (National Disability Insurance Agency, 2019) in Australia enabling funding for those with significant and permanent disability, the rigour of assessment and efficacy of interventions is paramount. Clinicians who have previously had little experience managing adults with $\mathrm{CP}$ are now providing much needed therapeutic interventions to this population. Study findings will provide a greater understanding of the impact of postural asymmetry and clinical measurement in non-ambulant adults with $\mathrm{CP}$, thus guiding interventions. Clinician knowledge of the lifespan care requirements is of extreme importance, ensuring adults with $\mathrm{CP}$ receive the best possible healthcare outcomes.

\section{Limitations}

A limitation of this study was a reliance on radiographs taken within one year of the GlofBS measurement, which assumes postural stability within the year or a very slow rate of change. However, given the often > 20-year history of abnormal forces impacting on postural symmetry in the cohort, we would argue a one-year timeframe was justified. The recently published National Institute for Health and Care Excellence (NICE) guidance on the management of adults with $\mathrm{CP}$ recognises the requirement for regular assessment, including the posture of adults with CP (Bromham et al., 2019). It has been well established that musculoskeletal complications are progressive (Tosi et al., 2009), yet due to a dearth of longitudinal studies, the rate of change is unknown. Until longitudinal evidence regarding the rate of change can be established, annual reviews as recommended by hip surveillance and NICE guidelines should be considered (Bromham et al., 2019; Wynter et al., 2014). It 
is for these reasons that a 12-month period between GlofBS measures and radiographs was considered appropriate.

Another limitation was the relatively high number of radiographs that were unable to be accurately read. This meant that missing data were evident for $17 \%$ (spine) and 10\% (hips) of the cohort, which may have influenced the interpretation of the relationship between radiographic and anthropometric data.

Figure 3 highlights that radiographs alone may not be adequate to document posture due to adults with complex disabilities and contractures being unable to achieve standardised positioning, variation in participant's position for radiographs, and potentially variation of expertise and knowledge amongst radiographers in attempting to obtain standardised alignment for valid and reliable measurements. As previously indicated, strategies for optimising the documented start position in radiographs in this population may enhance future radiographic quality and interpretation.

Participants in this study were non-ambulant adults with CP (GMFCS levels IV and V), managed by a specialist multidisciplinary team for complex medical issues and comorbidities, and findings may not be representative of those with less severe postural asymmetry (GMFCS Levels I-III).

Effective management of posture in non-ambulant adults with $C P$ is extremely challenging due to the combination of skeletal, muscular, and soft tissue distortion over a lifetime. Further, elements contributing to complex three-dimensional postural asymmetry of the thoracic cage, spine, pelvis, and hips, frequently encountered in adults with severe CP are difficult to quantify by standard two-dimensional methods, such as radiographs, alone.

Use of the GlofBS highlights the nature of postural asymmetry complementary to that obtained by radiographs, and may be useful in guiding interventions while ensuring relevant objectivity of clinical assessment is met for this challenging and complex group of adults. Inclusion of both radiographs and GlofBS could facilitate comprehensive clinical assessment for lifetime postural care of non-ambulant adults with CP.

\section{CONCLUSION}

This study showed there was minimal to no relationship between GlofBS measures and radiographic data for similar body areas in clinical measurement posture metrics in nonambulant adults with CP.

\section{KEY POINTS}

1. Non-ambulant adults with cerebral palsy (CP) are commonly affected by progressive secondary musculoskeletal issues which are challenging to manage.

2. The three-dimensional rotary nature of postural asymmetry is difficult to quantify by radiographs alone.

3. Postural asymmetry of the chest, pelvis, and windswept hips is objectively measured using Goldsmith Indices of Body Symmetry (GlofBS).

4. Use of radiographs and GlofBS facilitates lifespan care of non-ambulant adults with CP.

\section{DISCLOSURES}

No funding was obtained for this study. There are no conflicts of interest which may be perceived to interfere with or bias this study.

\section{PERIMISSIONS}

Ethical approval was gained from St. Vincent's Hospital, Melbourne, Human Research Ethics Committee (HREC/16/ SVHM/148). All participants (or their next of kin) provided informed consent.

\section{ACKNOWLEDGEMENTS}

The authorship team would like to acknowledge the contribution of Dr Kate Willoughby, Royal Children's Hospital, Melbourne, for the radiographical analysis component of the study.

\section{ADDRESS FOR CORRESPONDENCE}

Carlee Holmes, St. Vincent's Hospital, Melbourne, Australia.

Email: carlee.holmes@svha.org.au

\section{REFERENCES}

Ágústsson, A., Sveinsson, T., Pope, P., \& Rodby-Bousquet, E. (2019). Preferred posture in lying and its association with scoliosis and windswept hips in adults with cerebral palsy. Disability and Rehabilitation, 41(26), 3198-3202. https://doi.org/10.1080/09638288.2018.1492032

Benner, J. L., Noten, S., Limsakul, C., Van Der Slot, W. M. A., Stam, H. J., Selb, M., Van Den Berg-Emons, R. J. G., \& Roebroeck, M. E. (2019). Outcomes in adults with cerebral palsy: Systematic review using the International Classification of Functioning, Disability and Health. Developmental Medicine \& Child Neurology, 61(10), 1153-1161. https:// doi.org/10.1111/dmcn. 14247

Bromham, N., Dworzynski, K., Eunson, P., \& Fairhurst, C. (2019). Cerebral palsy in adults: Summary of NICE guidance. BMJ, 364, 1806. https://doi. org/10.1136/bmj.1806

Burns, F., Stewart, R., Reddihough, D., Scheinberg, A., Ooi, K., \& Graham, H. K. (2014). The cerebral palsy transition clinic: Administrative chore, clinical responsibility, or opportunity for audit and clinical research? Journal of Children's Orthopaedics, 8(3), 203-2013. https://doi.org/10.1007/s11832014-0569-0

Cobb, J. (1948). Outline for the study of scoliosis. American Academy of Orthopaedic Surgeons, 5, 261-275

Cohen, J. (1988). Statistical power analysis for the behavioral sciences (2nd ed.). Lawrence Erlbaum Associates.

Collis, F., Finger, E., Okerstrom, E., \& Owens, K. (2008). Review of transition of young adults clinics. Final report attachment 6: Literature review. https://www.aci.health.nsw.gov.au/_data/assets/pdf_file/0007/155779/ literaturereview.pdf

Farahani, N., Braun, A., Jutt, D., Huffman, T., Reder, N., Liu, Z., Yagi, Y., \& Pantanowitz, L. (2017).Three-dimensional imaging and scanning: Current and future applications for pathology. Journal of Pathology Informatics, 8, 36. https://doi.org/10.4103/jpi.jpi_32_17

Goldsmith, E., Golding, R. M., Garstang, R. A., \& MacRae, A. W. (1992) A technique to measure windswept deformity. Physiotherapy, 78(4), 235-242. https://doi.org/https://doi.org/10.1016/S0031-9406(10)61432-0

Goldsmith, E., \& Goldsmith, J. (2013). Goldsmith indices of body symmetry procedure (3rd ed.). https://www.simplestuffworks.com/wp-content/ uploads/2016/10/Goldsmith-Indices-of-Body-Symmetry-.pdf

Gstoettner, M., Sekyra, K., Walochnik, N., Winter, P., Wachter, R., \& Bach, C. M. (2007). Inter- and intraobserver reliability assessment of the Cobb angle: Manual versus digital measurement tools. European Spine Journal, 16(10), 1587-1592. https://doi.org/10.1007/s00586-007-0401-3 
Gudjonsdottir, B. B. S., \& Mercer, S. V. (1997). Hip and spine in children with cerebral palsy: Musculoskeletal development and clinical implications. Pediatric Physical Therapy, 9(4), 179-185. https://doi. org/10.1097/00001577-199700940-00005

Hägglund, G., Lauge-Pedersen, H., \& Wagner, P. (2007). Characteristics of children with hip displacement in cerebral palsy. BMC Musculoskeletal Disorders, 8, 101 (2007). https://doi.org/10.1186/1471-2474-8-101

Heidt, C., Hollander, K., Wawrzuta, J., Molesworth, C., Willoughby, K., Thomason, P., Khot, A., \& Graham, H. (2015). The radiological assessment of pelvic obliquity in cerebral palsy and the impact on hip development The Bone \& Joint Journal, 97B(10), 1435-1440. https://doi. org/10.1302/0301-620X.97B10.35390

Holmes, C., Brock, K., \& Morgan, P. (2018). Postural asymmetry in nonambulant adults with cerebral palsy: A scoping review. Disability and Rehabilitation, 41(9), 1079-1088. https://doi.org/10.1080/09638288.201 7.1422037

Holmes, C., Fredrickson, E., Brock, K., \& Morgan, P. (2020). The intra- and inter-rater reliability of the Goldsmith indices of body symmetry in nonambulant adults with cerebral palsy. Disability and Rehabilitation. Advance online publication. https://doi.org/10.1080/09638288.2019.1708979

Horimoto, Y., Osuda, Y., Takada, C., Tsugawa, S., Kozuka, N., Yoshida, S., Otani, T., \& Miwa, M. (2012). Thoracic deformity in the transverse plane among adults with severe cerebral palsy. Journal of Physical Therapy Science, 24(8), 763-766. https://doi.org/10.1589/jpts.24.763

Nana, A., Slater, G. J., Hopkins, W. G., Halson, S. L., Martin, D. T., West, N. P., \& Burke, L. M. (2016). Importance of standardized DXA protocol for assessing physique changes in athletes. International Journal of Sport Nutrition and Exercise Metabolism, 26(3), 259-267. https://doi. org/10.1123/ijsnem.2013-0111

National Disability Insurance Agency. (2019). National Disability Insurance Agency annual report 2018-19. https://www.ndis.gov.au/about-us/ publications/annual-report/annual-report-2018-19

Oda, Y., Takigawa, T., Sugimoto, Y., Tanaka, M., Akazawa, H., \& Ozaki, T.(2017). Scoliosis in patients with severe cerebral palsy: Three different courses in adolescents. Acta Medica Okayama, 71(2), 119-126. https:// doi.org/10.18926/AMO/54980

Palisano, R., Rosenbaum, P., Walter, S., Russell, D., Wood, E., \& Galuppi, B. (1997). Development and reliability of a system to classify gross motor function classification in children with cerebral palsy. Developmental Medicine and Child Neurology, 39(4), 214-223. https://doi. org/10.1111/j.1469-8749.1997.tb07414.x

Pruszczynski, B., Sees, J., \& Miller, F. (2016). Risk factors for hip displacement in children with cerebral palsy: Systematic review. Journal of Pediatric Orthopaedics, 36(8), 829-833. https://doi.org/10.1097/ BPO.0000000000000577

Public Health England. (2018). Postural Care and People with Learning Disabilities: Guidance. https://www.gov.uk/government/publications/ postural-care-services-making-reasonable-adjustments/postural-care-andpeople-with-learning-disabilities

Reimers, J. (1980). The stability of the hip joint in children. A radiological study of the results of muscle surgery in cerebral palsy. Acta Orthopaedica Scandinavica. Supplementum, 184, 1-100. https://doi.org/10.3109/ ort.1980.51.suppl-184.01
Robin, J., Graham, H. K., Baker, R., Selber, P., Simpson, P., Symons, S., \& Thomason, P. (2009). A classification system for hip disease in cerebral palsy. Developmental Medicine \& Child Neurology, 51(3), 183-192. https://doi.org/10.1111/j.1469-8749.2008.03129.x

Ryan, J. M., Peterson, M. D., Ryan, N., Smith, K. J., O'connell, N. E., Liverani, S., Anokye, N., Victor, C., \& Allen, E. (2019). Mortality due to cardiovascular disease, respiratory disease, and cancer in adults with cerebral palsy. Developmental Medicine \& Child Neurology, 61(8), 924-928. https://doi.org/10.1111/dmcn.14176

Sato, H., Kondo, M., Ojima, I., Fukasawa, H., \& Higuchi, S. (2016). Trunk deformity evaluation based on 3D measurements of front body surface landmarks in people with severe physical disabilities. Developmental Neurorehabilitation, 20(5), 280-286. https://doi.org/10.1080/17518423.2 016.1211188

Schmid, S., Buck, F. M., Böni, T., \& Farshad, M. (2016). Radiographic measurement error of the scoliotic curve angle depending on positioning of the patient and the side of scoliotic curve. European Spine Journal, 25(2), 379-384. https://doi.org/10.1007/s00586-015-4259-5

Shrader, M. W., Koenig, A. L., Falk, M., Belthur, M., \& Boan, C. (2017). An independent assessment of reliability of the Melbourne Cerebral Palsy Hip Classification System. Journal of Children's Orthopaedics, 11(5), 334-338. https://doi.org/10.1302/1863-2548.11.170077

Soo, B., Howard, J. J., Boyd, R. N., Reid, S. M., Lanigan, A., Wolfe, R., Reddihough, D., \& Graham, H. K. (2006). Hip displacement in cerebral palsy. The Journal of Bone \& Joint Surgery, 88(1), 121-129. https:// pubmed.ncbi.nlm.nih.gov/16391257/

Stewart, A. D., Klein, S., Young, J., Simpson, S., Lee, A. J., Harrild, K., Crockett, P., \& Benson, P. J. (2012). Body image, shape, and volumetric assessments using 3D whole body laser scanning and 2D digital photography in females with a diagnosed eating disorder: Preliminary novel findings. British Journal of Psychology, 103(2), 183-202. https://doi. org/10.1111/j.2044-8295.2011.02063.x

Suzuki, S., Kasahara, Y., Yamamoto, S., Seto, Y., Furukawa, K., \& Nishino, Y. (1993). Three-dimensional spinal deformity in scoliosis associated with cerebral palsy and with progressive muscular dystrophy. Spine, 18(15), 2290-2294. https://doi.org/10.1097/00007632-199311000-00026

Tasker, L. H., Shapcott, N. G., \& Holland, P. M. (2011). The use and validation of a laser scanner for computer aided design and manufacturing of wheelchair seating. Journal of Medical Engineering \& Technology, 35(6-7), 377-385. https://doi.org/10.3109/03091902.2011.601783

Tosi, L. L., Maher, N., Winslow Moore, D., Goldstein, M., \& Aisen, M. L. (2009). Adults with cerebral palsy: A workshop to define the challenges of treating and preventing secondary musculoskeletal and neuromuscular complications in this rapidly growing population. Developmental Medicine \& Child Neurology, 51(Supplement 4), 2-11. https://doi.org/10.1111/ j.1469-8749.2009.03462.x

World Physiotherapy. (2019). Advanced physical therapy practice. Policy statement. World Confederation for Physical Therapy. https://world.physio/ sites/default/files/2020-04/PS-2019-APTP_0.pdf

Wynter, M., Gibson, N., Kentish, M., Love, S. C., Thomason, P., Willoughby, K., \& Graham, H. K. (2014). Australian hip surveillance guidelines for children with cerebral palsy 2014. Australian Academy of Cerebral Palsy and Developmental Medicine. www.ausacpdm.org.au/professionals/hipsurveillance 